\title{
Porcupine inhibition: a novel disease-modifier target in arrhythmogenic cardiomyopathy
}

\author{
Jose M. Lopez-Ayala \\ Department of Cardiology, San Juan de Alicante General University Hospital, Alicante 03550, Spain. \\ Correspondence to: Dr. Jose M. Lopez-Ayala, Department of Cardiology, San Juan de Alicante General University Hospital, Ctra. \\ Nnal.332, Alicante 03550, Spain. E-mail: josemaria_lopezayala@yahoo.es

\begin{abstract}
How to cite this article: Lopez-Ayala JM. Porcupine inhibition: a novel disease-modifier target in arrhythmogenic
\end{abstract} \\ cardiomyopathy. J Cardiovasc Aging 2021;1:11. https://dx.doi.org/10.20517/jca.2021.14
}

Received: 27 Jul 2021 Accepted: 24 Aug 2021 First online: 27 Aug 2021

Academic Editor: Joshua Hare Copy Editor: Yue-Yue Zhang Production Editor: Yue-Yue Zhang

Arrhythmogenic cardiomyopathy is a common cause of ventricular arrhythmias and sudden death in young people and athletes. The pathologic hallmark of this genetically determined disease is myocardial necrosis and fibrofatty replacement, which constitutes a proarrhythmic substrate. The umbrella term arrhythmogenic cardiomyopathy encompasses three distinctive phenotypes: the classic arrhythmogenic right ventricular cardiomyopathy, left-dominant arrhythmogenic cardiomyopathy, and biventricular cardiomyopathy ${ }^{[1]}$. The initial genetic studies identified pathogenic variants in desmosomal genes in up to fifty percent of affected individuals, and pathogenic mutations in other proteins localized or interconnected with the intercalated disks were subsequently identified ${ }^{[2]}$.

Previous studies have demonstrated the pivotal role of the canonical Wnt/ $\beta$-catenin pathway in the development of the disease in a heterozygous desmoplakin mouse model. The complexity of the intercellular junction, not only as a cell-to-cell anchor, but also as an orchestrator of multiple downstream intracellular pathways was then revealed ${ }^{[3]}$. The ubiquitous canonical Wnt/ $\beta$-catenin pathway is central in the regulation of myocardial fibrosis and adipogenesis. In the paper published by Cheedipudi et al. ${ }^{[4]}$, WNT974, a Porcupine inhibitor in the canonical Wnt/ $\beta$-catenin pathway attenuates the expression of the arrhythmogenic cardiomyopathy in Myh6-Cre:Dsp ${ }^{W / F}$ mice. While previous research had mainly focused on the inhibition of the degradation of $\beta$-catenin (mainly targeting GSK-3 $\beta$ ), this research targets the initial step of the pathway, blocking the critical acetylation of Wnt molecules. The treatment with WNT974 at an early

The Author(s) 2021. Open Access This article is licensed under a Creative Commons Attribution 4.0 International License (https://creativecommons.org/licenses/by/4.0/), which permits unrestricted use, sharing, adaptation, distribution and reproduction in any medium or format, for any purpose, even commercially, as long as you give appropriate credit to the original author(s) and the source, provide a link to the Creative Commons license, and indicate if changes were made. 


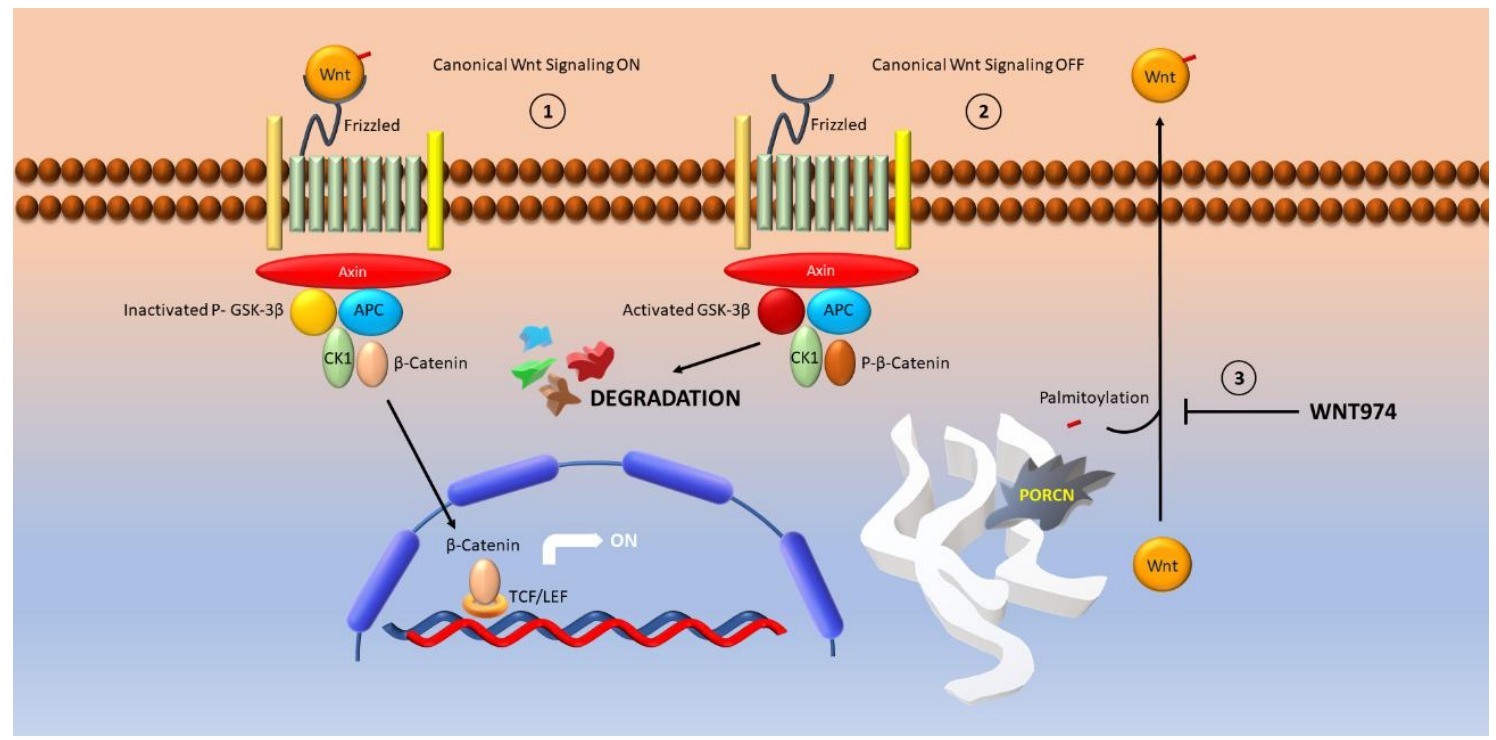

Figure 1. Canonical Wnt pathway in a cardiomyocyte of a Myh6-Cre-Dsp ${ }^{\mathrm{W} / \mathrm{F}}$ mouse model. (1) Upon activation, $\beta$-catenin translocates to the nucleus. Its interaction with TCF/LEF activates the expression of a myriad of genes involved in cardiac fibrosis. (2) The inactivation of the canonical Wnt pathway leads $\beta$-catenin to proteasomal degradation. (3) Porcupine, a post-translational enzyme localized to the endoplasmic reticulum, palmitoylates Wnt ligands for subsequent transmembrane transport and secretion to the extracellular space. WNT974, a Porcupine inhibitor, blocks this key step thereby suppressing the canonical pathway central to the pathogenesis of arrhythmogenic cardiomyopathy.

stage (3-month-old mice) before the development of an overt phenotype, was found to downregulate some genes critical to the myocardial fibrotic replacement [Figure 1]. These important findings were corroborated in the gross histology where significant differences in intramyocardial fibrosis and cardiomyocyte apoptosis were observed. Interestingly, the differences in the left ventricular diameters and ejection fraction between groups were remarkable. Downregulation of genes encoding natriuretic peptides (NPPA and NPPB) was also reported.

Arrhythmogenic cardiomyopathy, as with other cardiomyopathies, shows an incomplete penetrance and a variable clinical expression. In this regard, the identification of the genotype-positive/phenotype-negative individuals who will develop the disease or suffer sinister arrhythmias is paramount. Hence, the importance of targeting the disease at a molecular level during the "concealed phase" thereby preventing its progression. This is the strongest point of this research. It is worth pointing out the wide spectrum of phenotypes labeled as arrhythmogenic cardiomyopathy with different genetic backgrounds. The question of whether these findings would be also observed in in vitro and in vivo models bearing nonsense, frameshift, or missense mutations will require further research. The use of gene-editing tools such as CRISPR-Cas9 should be key to generate models that closely recapitulate the different human phenotypes of the diseas $\mathrm{e}^{[5]}$. It has been described that other pathways are also affected as a consequence of the disruption of the intercellular junctions. The suppression of Hippo, a pathway closely interconnected with the canonical Wnt/ $\beta$-catenin pathway, activates the expression of adipogenic genes ${ }^{[6]}$. In view of the complex interconnections between pathways, the simultaneous blockade of the canonical Wnt/ $\beta$-catenin and Hippo pathways arises as an attractive strategy.

It is important to note the discrepant results between experimental studies targeting the canonical Wnt/ $\beta$ catenin pathway; while the canonical pathway is suppressed in tissue samples of patients diagnosed with arrhythmogenic cardiomyopathy and in vitro mutant cardiomyocytes, its pharmacological suppression 
exerts a beneficial effect on fibrosis, apoptosis, and cardiac function in animal models of ischemic heart disease $\mathrm{e}^{[7]}$. The results of the paper published by Cheedipudi et al. ${ }^{[4]}$ support the latter. A possible explanation for this paradox may lie in intracellular compensatory mechanisms triggered by the remodeling of the intercalated disks and other stressing factors ${ }^{[8]}$. One may hypothesize that the association between canonical Wnt/ $\beta$-catenin inhibition and arrhythmogenic cardiomyopathy would not, therefore, be causative but a consequence of the disease.

In conclusion, the inhibition of canonical Wnt/ $\beta$-catenin with the Porcupine inhibitor WNT974 arises as a promising disease-modifying strategy in the early stages of arrhythmogenic cardiomyopathy. Further research will be required to determine its effect on overt phenotypes. The understanding of the molecular basis of arrhythmogenic cardiomyopathy is instrumental for bench-to-bed translational research and further personalized treatments.

\section{DECLARATIONS}

\section{Authors' contributions}

The author contributed solely to the article.

\section{Availability of data and materials}

Not applicable.

\section{Financial support and sponsorship}

None.

\section{Conflicts of interest}

The author declared that there are no conflicts of interest.

\section{Ethical approval and consent to participate}

Not applicable.

\section{Consent for publication}

Not applicable.

\section{Copyright}

(c) The Author(s) 2021.

\section{REFERENCES}

1. Corrado D, Perazzolo Marra M, Zorzi A, et al. Diagnosis of arrhythmogenic cardiomyopathy: The Padua criteria. Int $J$ Cardiol 2020;319:106-14. DOI PubMed

2. Austin KM, Trembley MA, Chandler SF, et al. Molecular mechanisms of arrhythmogenic cardiomyopathy. Nat Rev Cardiol 2019;16:519-37. DOI PubMed PMC

3. Garcia-Gras E, Lombardi R, Giocondo MJ, et al. Suppression of canonical Wnt/beta-catenin signaling by nuclear plakoglobin recapitulates phenotype of arrhythmogenic right ventricular cardiomyopathy. J Clin Invest 2006;116:2012-21. DOI PubMed PMC

4. Cheedipudi SM, Fan S, Rouhi L, Marian AJ. Pharmacological suppression of the WNT signaling pathway attenuates age-dependent expression of the phenotype in a mouse model of arrhythmogenic cardiomyopathy. J Cardiovasc Aging 2021;1:3. DOI

5. Musunuru K. Genome Editing: The recent history and perspective in cardiovascular diseases. J Am Coll Cardiol 2017;70:2808-21. DOI PubMed PMC

6. Rouhi L, Fan S, Cheedipudi SM, et al. The EP300/TP53 pathway, a suppressor of the Hippo and canonical WNT pathways, is activated in human hearts with arrhythmogenic cardiomyopathy in the absence of overt heart failure. Cardiovasc Res 2021;cvab197. DOI PubMed

7. Daskalopoulos EP, Blankesteijn WM. Effect of interventions in WNT signaling on healing of cardiac injury: a systematic review. Cells 2021;10:207. DOI PubMed PMC

8. Manolagas SC, Almeida M. Gone with the Wnts: beta-catenin, T-cell factor, forkhead box O, and oxidative stress in age-dependent diseases of bone, lipid, and glucose metabolism. Mol Endocrinol 2007;21:2605-14. DOI PubMed 